\title{
A flexible and versatile strategy for the covalent immobilization of chiral catalysts based on pyridinebis(oxazoline) ligands
}

Alfonso Cornejo, ${ }^{a}$ José M. Fraile, ${ }^{b}$ José I. García, ${ }^{b}$ María J. Gil, ${ }^{a}$ Santiago V. Luis, ${ }^{c}$ Víctor Martínez-Merino $^{a_{*}}$ and José A. Mayoral ${ }^{b_{*}}$

${ }^{a}$ Dep. Química Aplicada, Universidad Pública de Navarra, E-31006 Pamplona, Spain. Email: merino@unavarra.es

${ }^{b}$ Dep. Química Orgánica, Instituto de Ciencia de Materiales de Aragón, Universidad de Zaragoza-C.S.I.C., E-50009 Zaragoza, Spain.E-mail: mayoral@unizar.es

${ }^{c}$ Dep. Química Inorgánica y Orgánica, E.S.T.C.E., Universidad Jaume I, E-12080 Castellón, Spain

\section{Content index}

Page S-2: $\quad$ General experimental procedure.

Page S-3: $\quad$ Table S1 containing the B3LYP/SDD-6-31G* energies, enthalpies and Gibbs free energies of ethylene, p-cymene, and the pybox- $\mathrm{RuCl}_{2}$, pybox- $\mathrm{RuCl}_{2}$-ethylene and pybox- $\mathrm{RuCl}_{2}$-p-cymene complexes.

Pages S-3 to S-5: Cartesian coordinates of the pybox- $\mathrm{RuCl}_{2}$, pybox- $\mathrm{RuCl}_{2}$-ethylene and pybox- $\mathrm{RuCl}_{2}$-p-cymene complexes, optimized at the B3LYP/SDD-6$31 G^{*}$ theoretical level. 
General. All reactions were carried out under an atmosphere of dry nitrogen using flame-dried glassware and freshly distilled dry solvents. All the chromatographic separations were performed using silica gel (230-400 mesh). ${ }^{1} \mathrm{H}$ NMR spectra were recorded on a $200 \mathrm{MHz}$ spectrometer with TMS as internal standard. Chemical shifts are reported in ppm and coupling constants in Hz. Ruthenium analyses were carried out by plasma emission spectroscopy. Results of the cyclopropanation reactions were determined by gas chromatography with an FID detector; helium as carrier gas, 20 p.s.i.; injector temperature: $230{ }^{\circ} \mathrm{C}$; detector temperature: $250{ }^{\circ} \mathrm{C}$. The synthesis of the monomer 6 and the brominated intermediates 2a, 3a, 4a and 5a was described elsewhere. ${ }^{5}$ Chlorinated intermediates $\mathbf{2 b}, \mathbf{3 b}, \mathbf{4} \mathbf{b}$ and $\mathbf{5 b}$ were prepared by the method of Nishiyama et al. ${ }^{11}$ with only slight modifications. 
Table S1. B3LYP/SDD-6-31G* energies, enthalpies and Gibbs free energies of the structures calculated in this work.

$\begin{array}{rrr}\text { E(OK) } & \text { H(298K) } & \text { G(298K) } \\ -1755,901743 & -1755,663214 & -1755,730766 \\ -389,511161 & -389,286541 & -389,334965 \\ -78,587457 & -78,532246 & -78,557763 \\ & & \\ -2145,411078 & -2144,946578 & -2145,040525 \\ -1834,529525 & -1834,232480 & -1834,30489\end{array}$

pybox-RuCl2

\begin{tabular}{|c|c|c|c|}
\hline $\mathrm{Ru}$ & ๑. $000 \odot ० 8$ & $-\odot .958888$ & $\odot .000469$ \\
\hline $\mathrm{Cl}$ & $\odot .000114$ & -1.394125 & 2.378722 \\
\hline $\mathrm{Cl}$ & ๑.๑०๑९32 & -1.397739 & -2.377121 \\
\hline 0 & -3.583894 & 1.095035 & -0.000642 \\
\hline 0 & 3.583858 & 1.095108 & $-\odot .0 \odot \odot 72 \odot$ \\
\hline $\mathrm{N}$ & $-\odot .0 \odot \odot \odot 19$ & $\odot .945660$ & $-\odot .0 \odot \odot 231$ \\
\hline $\mathrm{N}$ & -2.050241 & $-\odot .567878$ & $\odot .00019 \odot$ \\
\hline $\mathrm{N}$ & 2.050216 & $-\odot .567816$ & ๑.0๑๑161 \\
\hline C & -1.186619 & 1.633893 & $-0.00 \odot 410$ \\
\hline C & -1.209159 & 3.025343 & $-\odot .0 \odot \odot 791$ \\
\hline C & $-\odot .0 \odot \odot \odot 68$ & 3.727752 & -0.000983 \\
\hline C & 1.209053 & 3.025383 & $-\odot .0 \odot \odot 8 \odot 9$ \\
\hline C & 1.186562 & 1.633935 & -0.000432 \\
\hline C & -2.299583 & $\odot .704448$ & -0.000234 \\
\hline C & -3.330495 & -1.282643 & 0.000101 \\
\hline C & -4.376516 & -0.135524 & $-\odot .0 \odot \odot 762$ \\
\hline C & 2.299550 & 0.704515 & $-\odot . \odot \odot \odot 274$ \\
\hline C & 3.330465 & -1.282580 & 0.000008 \\
\hline C & 4.376486 & -0.135460 & $-\odot .000695$ \\
\hline $\mathrm{H}$ & -2.164294 & 3.539114 & $-\odot .0 \odot \odot 927$ \\
\hline $\mathrm{H}$ & - ๑. .๑००८८ & 4.812277 & $-\odot .001260$ \\
\hline $\mathrm{H}$ & 2.164168 & 3.539190 & $-\odot .000958$ \\
\hline $\mathrm{H}$ & -3.391905 & -1.917574 & 0.889229 \\
\hline $\mathrm{H}$ & -3.391312 & -1.918404 & $-\odot .888468$ \\
\hline $\mathrm{H}$ & -5.005731 & -0.117620 & 0.891764 \\
\hline $\mathrm{H}$ & -5.004723 & $-\odot .118087$ & -0.894015 \\
\hline $\mathrm{H}$ & 3.391874 & -1.917612 & 0.889065 \\
\hline $\mathrm{H}$ & 3.391299 & -1.918225 & -0.888644 \\
\hline $\mathrm{H}$ & 5.005540 & $-\odot .117547$ & 0.891944 \\
\hline $\mathrm{H}$ & 5.004851 & -0.118018 & $-\odot .89383$ \\
\hline
\end{tabular}


pybox-RuCl2-ethylene

\begin{tabular}{|c|c|c|c|}
\hline 24 & 3.7000 & 0.7500 & -2.4970 \\
\hline & 2.3090 & -0.3140 & -4.2420 \\
\hline $\mathrm{Cl}$ & 5.0940 & 1.7480 & -0.7160 \\
\hline & 6.5500 & 1.7970 & -5.3540 \\
\hline & $\odot .6640$ & 3.4980 & -1.7100 \\
\hline & 3.6120 & 2.5230 & -3.4650 \\
\hline & 5.3640 & $\odot .6920$ & -3.7760 \\
\hline & 1.9930 & 1.6670 & -1.6870 \\
\hline & 4.5500 & 2.8360 & -4.3880 \\
\hline C & 4.5220 & 4.0480 & -5.0750 \\
\hline & 3.4930 & 4.9500 & -4.7920 \\
\hline & 2.5240 & 4.6260 & -3.8380 \\
\hline C & 2.6160 & 3.3950 & -3.1900 \\
\hline & 5.5100 & 1.7490 & -4.5090 \\
\hline c & 6.4610 & -0.2280 & -4.0950 \\
\hline C & 7.2630 & 0.5320 & -5.1840 \\
\hline C & 1.7370 & 2.8400 & -2.1720 \\
\hline C & $\odot .9600$ & 1.3600 & $-\odot .6920$ \\
\hline & 0.0540 & 2.6180 & -0.7140 \\
\hline c & 4.3630 & -1.2660 & -1.8420 \\
\hline C & 3.2180 & -0.9340 & -1.1320 \\
\hline $\mathrm{H}$ & 5.2880 & 4.2700 & -5.8090 \\
\hline $\mathrm{H}$ & 3.4460 & 5.9020 & -5.3110 \\
\hline $\mathrm{H}$ & 1.7130 & 5.3040 & -3.5950 \\
\hline H & $6.049 \odot$ & -1.1750 & -4.4550 \\
\hline $\mathrm{H}$ & 7.0470 & -0.4210 & -3.1920 \\
\hline $\mathrm{H}$ & 7.2680 & 0.0280 & -6.1530 \\
\hline H & 8.2860 & 0.7710 & -4.8870 \\
\hline H & 0.4300 & 0.4500 & -0.9880 \\
\hline $\mathrm{H}$ & 1.4300 & 1.1910 & 0.2810 \\
\hline $\mathrm{H}$ & -0.9680 & 2.4160 & -1.0400 \\
\hline $\mathrm{H}$ & 0.0370 & 3.1610 & 0.2340 \\
\hline 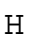 & 4.2940 & -1.9200 & -2.7070 \\
\hline п & 5.3400 & -1.1460 & -1.3830 \\
\hline & 2.2540 & -1.3290 & -1.4410 \\
\hline $\mathrm{H}$ & & & \\
\hline
\end{tabular}


pybox-RuCl2-p-cymene

\begin{tabular}{|c|c|c|c|}
\hline Ru & 0.390613 & $-\odot .151984$ & ๑.218725 \\
\hline $\mathrm{Cl}$ & $\odot .952666$ & 0.136236 & 2.603043 \\
\hline $\mathrm{Cl}$ & $-\odot .195270$ & -0.526274 & -2.147221 \\
\hline 0 & 1.101826 & 3.841755 & $-\odot .556250$ \\
\hline 0 & 3.460057 & -2.900183 & $-\odot .161195$ \\
\hline $\mathrm{N}$ & 2.153314 & 0.428517 & -0.294251 \\
\hline $\mathrm{N}$ & 0.082503 & 1.898556 & $-\odot .0 \odot 4512$ \\
\hline $\mathrm{N}$ & 1.432687 & -1.970323 & 0.219710 \\
\hline C & 2.385701 & 1.747923 & $-\odot .543119$ \\
\hline C & 3.649581 & 2.198042 & $-\odot .917656$ \\
\hline C & 4.688772 & 1.270715 & -1.036711 \\
\hline C & 4.446738 & $-\odot .082359$ & -0.783105 \\
\hline C & 3.163224 & -0.477145 & -0.412888 \\
\hline C & 1.163631 & 2.514791 & $-\odot .361753$ \\
\hline C & -0.992392 & 2.891424 & 0.095410 \\
\hline C & $-\odot .288875$ & 4.217482 & -0.298278 \\
\hline C & 2.674919 & -1.812562 & $-\odot .109026$ \\
\hline C & 1.207453 & -3.401055 & 0.449349 \\
\hline C & 2.610950 & -4.025177 & $\odot .227608$ \\
\hline $\mathrm{H}$ & 3.804196 & 3.253938 & -1.109784 \\
\hline $\mathrm{H}$ & 5.680827 & 1.600416 & -1.326495 \\
\hline $\mathrm{H}$ & 5.230475 & $-\odot .826812$ & $-\odot .868316$ \\
\hline $\mathrm{H}$ & -1.386189 & 2.899465 & 1.115692 \\
\hline $\mathrm{H}$ & -1.804507 & 2.614012 & $-\odot .582386$ \\
\hline $\mathrm{H}$ & $-\odot .281871$ & 4.963141 & 0.499712 \\
\hline $\mathrm{H}$ & -0.680529 & 4.663955 & -1.215116 \\
\hline $\mathrm{H}$ & ๑.829827 & -3.555731 & 1.464648 \\
\hline $\mathrm{H}$ & 0.460469 & -3.768085 & $-\odot .262105$ \\
\hline $\mathrm{H}$ & 3.040418 & -4.460511 & 1.132891 \\
\hline $\mathrm{H}$ & 2.643779 & -4.755367 & $-\odot .583531$ \\
\hline C & -4.147248 & 0.417988 & 0.202609 \\
\hline C & -2.161066 & -0.961467 & 1.570282 \\
\hline C & -3.831143 & 0.759635 & 1.524386 \\
\hline C & -3.485005 & -0.616723 & -0.461049 \\
\hline C & -2.482146 & -1.302208 & 0.252720 \\
\hline C & -2.842095 & ๑.080432 & 2.236478 \\
\hline C & -2.530760 & 0.411387 & 3.675007 \\
\hline $\mathrm{H}$ & -3.076451 & 1.301963 & 4.005478 \\
\hline $\mathrm{H}$ & -1.456773 & $\odot .581686$ & 3.806890 \\
\hline $\mathrm{H}$ & -2.813562 & -0.415340 & 4.340208 \\
\hline C & -3.862108 & -1.043906 & -1.873259 \\
\hline $\mathrm{H}$ & -3.086576 & -1.740596 & -2.212917 \\
\hline C & -3.875077 & 0.129510 & -2.868286 \\
\hline $\mathrm{H}$ & -4.113221 & -0.231199 & -3.876211 \\
\hline $\mathrm{H}$ & -2.892888 & 0.610111 & -2.903526 \\
\hline $\mathrm{H}$ & -4.629988 & ๑.880444 & -2.602772 \\
\hline C & -5.211862 & -1.790472 & -1.878103 \\
\hline $\mathrm{H}$ & -5.451481 & -2.150472 & -2.886178 \\
\hline $\mathrm{H}$ & -6.028219 & -1.132476 & -1.554706 \\
\hline $\mathrm{H}$ & -5.194330 & -2.654013 & -1.203232 \\
\hline $\mathrm{H}$ & -4.939595 & 0.964734 & $-\odot .303816$ \\
\hline $\mathrm{H}$ & -4.384561 & 1.559333 & 2.013435 \\
\hline $\mathrm{H}$ & -1.420379 & -1.539194 & 2.116382 \\
\hline $\mathrm{H}$ & -1.973313 & -2.132811 & -0.227910 \\
\hline
\end{tabular}

